\title{
Distinct pathogenesis in nonsystemic vasculitic neuropathy and microscopic polyangiitis OPEN
}

Mie Takahashi, MD

Haruki Koike, MD, PhD

Shohei Ikeda, MD

Yuichi Kawagashira, MD,

$\mathrm{PhD}$

Masahiro Iijima, MD,

$\mathrm{PhD}$

Atsushi Hashizume, MD, $\mathrm{PhD}$

Masahisa Katsuno, MD, $\mathrm{PhD}$

Gen Sobue, MD, PhD

Correspondence to

Dr. Koike:

koike-haruki@med.nagoya-u.ac.jp

or Dr. Sobue:

sobueg@med.nagoya-u.ac.jp
Supplemental data at Neurology.org/nn

\section{ABSTRACT}

Objective: To investigate the mechanisms of vasculitis in nonsystemic vasculitic neuropathy (NSVN) and microscopic polyangiitis (MPA), focusing on complement- and antineutrophil cytoplasmic antibody (ANCA)-associated pathogenesis.

Methods: Sural nerve biopsy specimens taken from twenty-four patients with NSVN and 37 with MPA-associated neuropathy (MPAN) were examined. Twenty-two patients in the MPAN group tested positive for ANCA.

Results: Immunostaining for complement component C3d deposition showed more frequent positive staining of epineurial small vessels in NSVN than in MPAN ( $p=0.002)$. The percentages of C3d-positive blood vessels were higher in the NSVN group than those in the ANCA-positive MPAN and ANCA-negative MPAN groups ( $p=0.002$ and $p=0.009$, respectively). Attachment of neutrophils to the endothelial cells of epineurial small vessels was frequently observed in the MPAN groups, irrespective of the presence or absence of ANCA, but was scarce in the NSVN group. Immunohistochemistry using antimyeloperoxidase (MPO) antibodies revealed that the number of MPO-positive cells attached to the endothelial cells of epineurial vessels was lower in the NSVN group than that in the ANCA-positive MPAN and ANCA-negative MPAN groups ( $p<$ 0.001 and $p=0.011$, respectively).

Conclusions: NSVN and MPA have distinct mechanisms of vasculitis. In MPA, the attachment of neutrophils to vascular endothelial cells seems to be an initial lesion of vasculitis, regardless of the presence or absence of ANCA. Complement participated in the pathogenesis of vasculitis in NSVN. Neurol Neuroimmunol Neuroinflamm 2017;4:e407; doi: 10.1212/NXI.0000000000000407

\section{GLOSSARY}

ANCA $=$ antineutrophil cytoplasmic antibody; $\mathbf{C R P}=$ C-reactive protein; ESR $=$ erythrocyte sedimentation rate; $\mathbf{M P A}=$ microscopic polyangiitis; $\mathbf{M P O}=$ myeloperoxidase; $\mathbf{M P A N}=$ MPA-associated neuropathy; $\mathbf{N S V N}=$ nonsystemic vasculitic neuropathy; PBS = phosphate-buffered saline; PR3 = proteinase 3.

Vasculitides are diseases caused by the inflammation of vessels. They affect a variety of vessels, ranging from the aorta to capillaries in a variety of organs. ${ }^{1}$ The lungs, heart, kidneys, gastrointestinal tract, skin, and nervous system are frequently involved in vasculitides. ${ }^{2,3}$ In the peripheral nervous system, vasculitis usually occurs in small vessels located in the epineurium, leading to ischemia and subsequent axonal degeneration. ${ }^{4,5}$ Hence, vasculitides affecting small vessels, such as antineutrophil cytoplasmic antibody (ANCA)-associated vasculitis, frequently affect the peripheral nervous system. ${ }^{2,3}$ For example, microscopic polyangiitis (MPA) and eosinophilic granulomatosis with polyangiitis (previously known as Churg-Strauss syndrome) affect the peripheral nervous system in up to $60 \%-80 \%$ of patients, respectively. ${ }^{2,3,6}$ In addition to these ANCA-associated vasculitides, ANCA-negative vasculitis confined to the peripheral nervous system has also been reported in neurology and constitutes one of the major clinical categories of vasculitic neuropathy. ${ }^{7-13}$ Although this disease has been called nonsystemic

From the Department of Neurology (M.T., H.K., S.I., Y.K., M.I., A.H., M.K., G.S.), and Research Division of Dementia and Neurodegenerative Disease (G.S.), Nagoya University Graduate School of Medicine, Japan.

Funding information and disclosures are provided at the end of the article. Go to Neurology.org/nn for full disclosure forms.

This is an open access article distributed under the terms of the Creative Commons Attribution-NonCommercial-NoDerivatives License 4.0 (CC BY-NC-ND), which permits downloading and sharing the work provided it is properly cited. The work cannot be changed in any way or used commercially without permission from the journal. 
vasculitic neuropathy (NSVN), ${ }^{8}$ no consensus exists as to whether NSVN is an independent nosological entity or occurs on a continuum of neuropathies associated with systemic vasculitides. In particular, the distinction between NSVN and MPA is not well defined because the current definition of the latter includes ANCA-negative cases. ${ }^{1,5,11,14}$

In this study, we examined sural nerve biopsy samples taken from patients with NSVN and MPA, with a particular focus on ANCA- and complement-associated pathogenesis of vasculitis.

METHODS Patients. We retrospectively investigated patients with pathologically confirmed NSVN and MPA-associated neuropathy (MPAN) who were referred for diagnostic sural nerve biopsy to Nagoya University Graduate School of Medicine between 1997 and 2015. NSVN was diagnosed according to the criteria of the "Peripheral Nerve Society Guidelines for Nonsystemic Vasculitic Neuropathy." ${ }^{13}$ In the NSVN group, symptoms of vasculitis were confined solely to the peripheral nerves, with no evidence of other organ involvement. Laboratory data also indicated the absence of any other underlying disease, and the erythrocyte sedimentation rate (ESR) was less than $100 \mathrm{~mm} / \mathrm{h} .{ }^{13}$ The presence of ANCA was an exclusion criterion for the NSVN group. Inclusion criteria for the MPAN group were based on the definition in the "2012 Revised International Chapel Hill Consensus Conference Nomenclature of Vasculitis (CHCC2012)." Involvement of organs other than the nervous system, such as the lungs, skin, heart, gastrointestinal tract, and kidneys, or a positive titer for ANCA was mandatory for inclusion in the MPAN group. ANCAs directed against myeloperoxidase (MPO) and proteinase 3 (PR3) were screened by ELISA. The presence or absence of vascular immune deposits was not considered at the time of inclusion.

Subjects meeting criteria for pathologically definite or probable vasculitic neuropathy were included in this study. ${ }^{13}$ Based on these criteria, 24 patients with NSVN and 37 with MPAN were included. In the ANCA-positive MPAN group, 14 patients had pathologically definite and 8 had probable vasculitic neuropathy. The corresponding diagnoses were 7 and 8 in the ANCA-negative MPAN group and 11 and 13 in the NSVN group. Eleven patients in the NSVN group and 4 in the MPAN group had been included in a previous study. ${ }^{11}$ Patients with other diseases that may cause vasculitis, such as eosinophilic granulomatosis with polyangiitis, granulomatosis with polyangiitis, malignancyassociated vasculitis, or connective tissue disease-associated vasculitis, were excluded. ${ }^{1,13}$ Patients in the NSVN group were followed up for $4.8 \pm 4.7$ (mean $\pm \mathrm{SD}$ ) years after diagnosis to confirm that their vasculitis was nonsystemic. Twenty-two of the 37 patients $(60 \%)$ in the MPAN group were positive for MPO-ANCA; none were positive for PR3-ANCA. All ANCAnegative MPAN patients had findings suggestive of extraneural involvement. Because these ANCA-negative MPAN patients are particularly controversial from the viewpoint of the distinction from NSVN, subgroups of ANCA-positive MPAN and ANCAnegative MPAN were separately assessed.

Standard protocol approvals, registrations, and patient consent. This study conformed to the Ethical Guidelines for Medical and Health Research Involving Human Subjects endorsed by the Japanese government and was approved by the Ethics Committees of Nagoya University Graduate School of Medicine, and all patients provided written informed consent.

Pathologic assessment of sural nerve biopsy specimens. Histopathologic examinations of the sural nerve were performed on all patients under local anesthesia before the initiation of immunotherapy. ${ }^{15,16}$ The specimens were divided into 2 portions. One portion was fixed in $2.5 \%$ glutaraldehyde in $0.125 \mathrm{M}$ cacodylate buffer and embedded in epoxy resin. The density of myelinated fibers was calculated in toluidine blue-stained semithin sections as described previously. ${ }^{15,17}$ For electron microscopy, epoxy resin-embedded specimens were cut into ultrathin transverse sections and stained with uranyl acetate and lead citrate. ${ }^{18-20}$ A part of the sample was processed for a teased-fiber study. ${ }^{21}$ The control values were based on a previous report. ${ }^{22}$ The second portion of the specimen was fixed in $10 \%$ formalin and embedded in paraffin. Sections were sliced using routine methods and then stained with hematoxylin and eosin and Masson trichrome. The investigators who evaluated the biopsies were masked to the clinical diagnosis.

Immunofluorescence and immunohistochemical studies. Immunofluorescence and immunohistochemistry were performed in all samples using serial $4-\mu \mathrm{m}$-thick sections from paraffinembedded specimens. To evaluate vascular complement deposition, indirect immunofluorescence was performed. ${ }^{18-20}$ Sections were deparaffinized and heated at $98^{\circ} \mathrm{C}$ for 10 minutes in citrate buffer for antibody retrieval $(\mathrm{pH}$ 6.0) and left to cool at room temperature for 10 minutes. Samples were then immersed in $0.3 \%$ hydrogen peroxide and washed 3 times with phosphatebuffered saline (PBS). A rabbit monoclonal antibody against complement component C3d (1:50; Abcam, Cambridge, United Kingdom) served as the primary antibody and was dissolved in blocking solution with goat serum and then incubated for 60 minutes at $4^{\circ} \mathrm{C}$. After washes with PBS, the sample was incubated for 3 hours at room temperature with Alexa Fluor donkey anti-rabbit $\operatorname{IgG}(\mathrm{H}+\mathrm{L}, 1: 1,000)$. The stained sections were examined and photographed using an optical microscope (FSX 100; Olympus, Tokyo, Japan).

To evaluate the location of MPO-positive polymorphonuclear leukocytes, immunohistochemistry was performed via immunoperoxidase staining. ${ }^{23}$ Sections were deparaffinized and heated at $98^{\circ} \mathrm{C}$ for 10 minutes in citrate buffer for antibody retrieval ( $\mathrm{pH} \mathrm{6.0)}$ and left to cool at room temperature for $10 \mathrm{mi}$ nutes. After immersion in $0.3 \%$ hydrogen peroxide, sections were washed 3 times with PBS. Primary antibodies (rabbit polyclonal anti-MPO; 1:100; Abcam) were dissolved in blocking solution containing goat serum and incubated for 60 minutes at $4^{\circ} \mathrm{C}$. After washes with PBS, the sections were incubated for 30 minutes at room temperature with anti-rabbit $\operatorname{IgG}$ conjugated to biotin, washed again with PBS, and incubated for 60 minutes at $4^{\circ} \mathrm{C}$ with an avidin-biotin complex.

All epineurial blood vessels in the complete transverse sections of the sural nerve were assessed for immunohistochemical and immunofluorescent studies in each case. The amount of MPOpositive cells attached to the endothelial cells of epineurial vessels was evaluated as the number of these cells per vessel. To identify the initial lesions that led to vasculitis, vessels that retained their morphology were included in the quantitative analyses.

Statistical analysis. Quantitative data are presented as mean \pm SD unless otherwise indicated. Statistical analyses were performed using $\chi^{2}$ tests or 1 -way analysis of variance to assess differences among all 3 groups. Subsequently, the Tukey-Kramer method was used as a post hoc test to identify significant differences 
between each pair of patient groups, if necessary. $p$ Values $<0.05$ were considered statistically significant.

RESULTS Characteristics of patients. The patient characteristics are summarized in table 1 and reported as medians and ranges in table e-1 at Neurology.org/nn. Although the age at biopsy seemed to be younger in the NSVN group than in the MPAN groups, the demographics and neuropathic features in the NSVN and MPAN groups were not significantly different. In the ANCA-positive MPAN group, findings suggestive of renal, pulmonary, cutaneous, and gastrointestinal tract involvement were noted in $32 \%, 41 \%$, $36 \%$, and $24 \%$ of patients, respectively. Two patients in the ANCA-positive group (9\%) had clinically isolated neuropathy, with no apparent involvement of other organs. In the ANCA-negative MPAN group, these proportions were $40 \%, 33 \%, 33 \%$, and $20 \%$, respectively. C-reactive protein (CRP), ESR, and white blood cell counts were higher in the

Table 1 Patient characteristics

\begin{tabular}{|c|c|c|c|c|c|}
\hline & $\begin{array}{l}\text { MPA (all cases) } \\
n=37\end{array}$ & $\begin{array}{l}\text { MPA (ANCA positive) } \\
n=22\end{array}$ & $\begin{array}{l}\text { MPA (ANCA negative) } \\
\mathrm{n}=15\end{array}$ & $\begin{array}{l}\text { NSVN } \\
n=24\end{array}$ & p Value ${ }^{a}$ \\
\hline Age at biopsy, y & $64.8 \pm 12.9$ & $63.5 \pm 14.9$ & $66.8 \pm 9.5$ & $58.0 \pm 16.5$ & NS \\
\hline Men/women & $20 / 17$ & $10 / 12$ & $10 / 5$ & $13 / 11$ & NS \\
\hline \multicolumn{6}{|l|}{ Initial symptom of neuropathy } \\
\hline Sensory disturbance & $32(87)$ & $19(86)$ & $13(87)$ & $21(88)$ & NS \\
\hline Muscle weakness & $5(14)$ & $3(14)$ & $2(13)$ & $3(13)$ & \\
\hline Duration of neuropathy, $\mathrm{mo}^{\mathrm{b}}$ & $4.8 \pm 4.5$ & $3.9 \pm 3.7$ & $6.2 \pm 5.3$ & $7.9 \pm 7.8$ & NS \\
\hline $\mathrm{CRP}, \mathrm{mg} / \mathrm{dL}$ & $8.9 \pm 7.4$ & $8.4 \pm 5.0$ & $9.6 \pm 10.2$ & $1.3 \pm 2.9$ & $<0.001$ \\
\hline $\mathrm{ESR}, \mathrm{mm} / \mathrm{h}$ & $64.0 \pm 37.9$ & $76.9 \pm 38.6$ & $49.2 \pm 32.4$ & $33.9 \pm 28.0$ & 0.002 \\
\hline White blood cell count, no. $/ \mathrm{mm}^{3}$ & $11,948 \pm 6,674$ & $10,959 \pm 4,586$ & $13,398 \pm 8,900$ & $7,351 \pm 3,602$ & 0.007 \\
\hline $\mathrm{C} 3, \mathrm{mg} / \mathrm{dL}$ & $112.9 \pm 31.4$ & $111.3 \pm 27.3$ & $115.3 \pm 37.3$ & $99.2 \pm 23.4$ & NS \\
\hline $\mathrm{C} 4, \mathrm{mg} / \mathrm{dL}$ & $28.6 \pm 20.2$ & $29.9 \pm 24.4$ & $26.8 \pm 13.0$ & $25.6 \pm 17.0$ & NS \\
\hline $\mathrm{CH} 50, \mathrm{U} / \mathrm{mL}$ & $42.4 \pm 15.1$ & $44.3 \pm 13.7$ & $42.5 \pm 18.8$ & $42.2 \pm 15.3$ & NS \\
\hline CSF protein, mg/dL & $36.9 \pm 16.9$ & $30.0 \pm 8.6$ & $39.5 \pm 19.9$ & $44.6 \pm 18.1$ & NS \\
\hline \multicolumn{6}{|l|}{ Nerve conduction studies $^{c}$} \\
\hline \multicolumn{6}{|l|}{ Median nerve } \\
\hline CMAP, mV & $5.1 \pm 3.5$ & $6.2 \pm 3.7$ & $3.4 \pm 2.2$ & $4.7 \pm 3.7$ & NS \\
\hline SNAP, $\mu \mathrm{V}$ & $13.4 \pm 13.4$ & $17.0 \pm 15.2$ & $7.7 \pm 7.3$ & $8.2 \pm 10.6$ & NS \\
\hline \multicolumn{6}{|l|}{ Tibial nerve } \\
\hline CMAP, mV & $2.1 \pm 3.6$ & $2.5 \pm 4.2$ & $1.6 \pm 2.7$ & $5.9 \pm 7.0$ & NS \\
\hline \multicolumn{6}{|l|}{ Sural nerve } \\
\hline SNAP, $\mu \mathrm{V}$ & $0.9 \pm 1.7$ & $0.8 \pm 1.8$ & $1.2 \pm 1.7$ & $2.4 \pm 4.8$ & NS \\
\hline \multicolumn{6}{|l|}{ Pathology of the sural nerve $e^{d}$} \\
\hline Total MFD, no. $/ \mathrm{mm}^{2}$ & $2,649 \pm 3,846$ & $2,619 \pm 4,364$ & $2,272 \pm 3,208$ & $2,455 \pm 3,073$ & NS \\
\hline Large MFD, no./mm² & $877 \pm 1,525$ & $968 \pm 1,814$ & $758 \pm 1,098$ & $686 \pm 996$ & NS \\
\hline Small MFD, no./mm² & $1,578 \pm 2,347$ & $1,651 \pm 2,570$ & $1,483 \pm 2,118$ & $1,769 \pm 2,200$ & NS \\
\hline \multicolumn{6}{|l|}{ Teased-fiber study } \\
\hline De/remyelination, \% & $1.2 \pm 1.6$ & $1.1 \pm 1.7$ & $1.5 \pm 1.5$ & $2.7 \pm 3.3$ & NS \\
\hline Axonal degeneration, \% & $70.5 \pm 33.2$ & $79.2 \pm 29.1$ & $58.6 \pm 36.0$ & $58.8 \pm 33.1$ & NS \\
\hline
\end{tabular}

Abbreviations: ANCA = antineutrophil cytoplasmic antibody; CMAP = compound muscle action potential; CRP = C-reactive protein; ESR = erythrocyte sedimentation rate; MFD = myelinated fiber density; MPA = microscopic polyangiitis; NS = not significant; NSVN = nonsystemic vasculitic neuropathy; SNAP = sensory nerve action potential.

Data are number $(\%)$ or mean \pm SD.

a One-way analysis of variance (ANCA-positive MPA, ANCA-negative MPA, and NSVN groups).

${ }^{b}$ Duration of neuropathy until sural nerve biopsy.

${ }^{\mathrm{c}}$ Control values for CMAP in the median nerve, SNAP in the median nerve, CMAP in the tibial nerve, and SNAP in the sural nerve are $8.2 \pm 2.9,28.0 \pm 11.5,11.8 \pm 3.5$, and $16.8 \pm 7.8$, respectively. ${ }^{16}$

${ }^{d}$ Control values for total MFD, large MFD, small MFD, de/remyelination, and axonal degeneration are 8,247 $\pm 737,3,129$

$\pm 642,5,118 \pm 429,8.6 \pm 6.7$, and $1.8 \pm 1.6$, respectively. $^{22}$ 
ANCA-positive MPAN and ANCA-negative MPAN groups than in the NSVN group.

Indices of sural nerve biopsy specimens, including the densities of myelinated fibers and the frequencies of segmental de/remyelination and axonal degeneration, were not significantly different among the 3 groups.

Complement deposition on epineurial vessels. We assessed the deposition of complement component C3d around epineurial vessels. Although epineurial vessels that exhibited signs of vasculitis, such as destruction of vessel walls accompanied by inflammatory cellular infiltration, showed some positive antiC3d antibody staining, some epineurial vessels with preserved morphology also stained positively (figure 1). When we assessed these vessels with preserved morphology, C3d deposition was more frequently observed in patients with NSVN than in those with MPAN $(23 \%$ of patients in the ANCA-positive MPAN group, $33 \%$ in the ANCA-negative MPAN group, and $71 \%$ in the NSVN group; $p=0.002$ ).

Figure 1 Representative images of complement component C3d immunofluorescence in sural nerve biopsy specimens
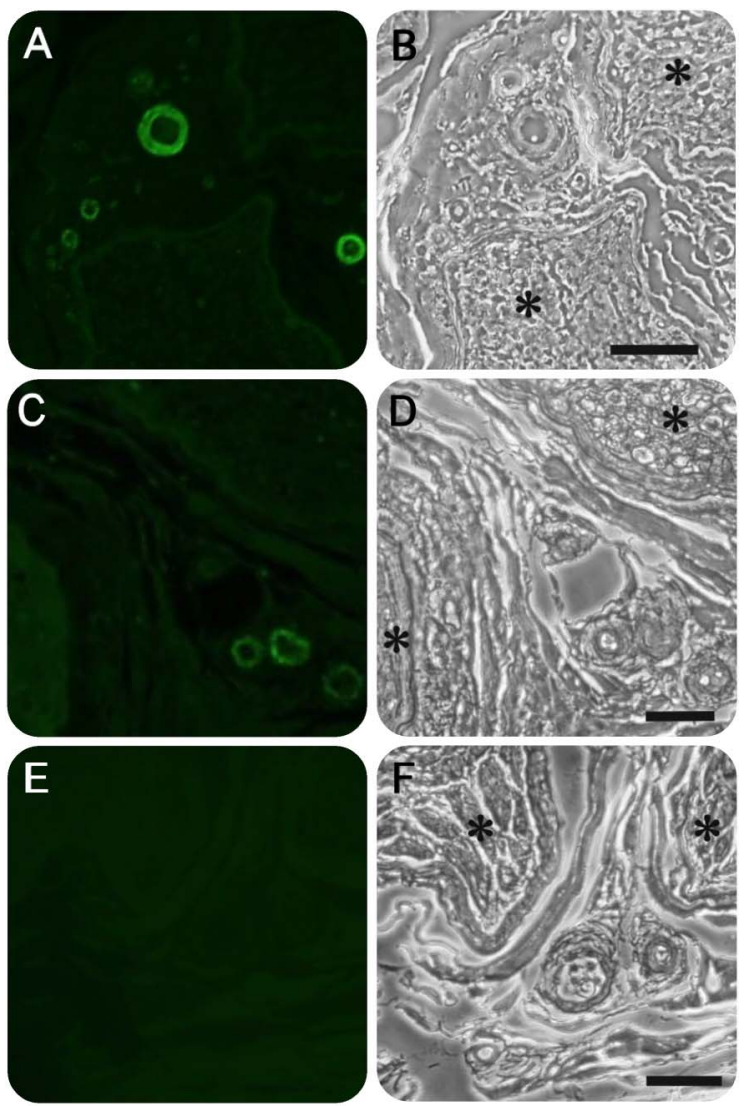

Photographs of nonsystemic vasculitic neuropathy (NSVN) (A to D) and microscopic polyangiitisassociated neuropathy (MPAN) (E and F). Epineurial small vessels were positively stained with antibodies against C3d in NSVN (A and C), whereas those in MPAN were not stained with these antibodies (E). Phase-contrast microscopy images of $A, C$, and $E$ are shown in $B, D$, and $F$, respectively. Nerve fascicles are indicated by asterisks in B, D, and F. Scale bars $=100 \mu \mathrm{m}$.
The percentages of C3d-positive epineurial blood vessels in the complete transverse sections of the sural nerve were $2.6 \pm 7.2$ in the ANCA-positive MPAN group, $3.1 \pm 5.2$ in the ANCA-negative MPAN group, and $15.7 \pm 18.0$ in the NSVN group $(p=$ 0.001 , figure 2 and table e-2). The diameters of these vessels were usually 30-70 $\mu \mathrm{m}$. Serum C3 levels were slightly (but not significantly) lower in NSVN with positive C3d deposition than in that with negative C3d deposition $(93 \pm 22 \mathrm{mg} / \mathrm{dL}$ vs $114 \pm 25 \mathrm{mg} / \mathrm{dL}$ ).

C3d deposition on epineurial vessels was observed in only 10 patients with MPAN, 5 in the ANCA-positive MPAN group and 5 in the ANCA-negative MPAN group. However, the intensity of immunostaining was much weaker in those cases than that in the NSVN cases, corresponding to the CHCC2012 definition of MPA. ${ }^{1}$ Concerning damage to organs other than the peripheral nervous system, 2 of these cases showed no substantial damage to other organs but were diagnosed as MPA because of positive ANCA. Seven other cases had 1 additionally damaged organ: lung in 1, kidney in

\section{Figure 2 The percentage of complement component C3d-positive epineurial blood vessels}
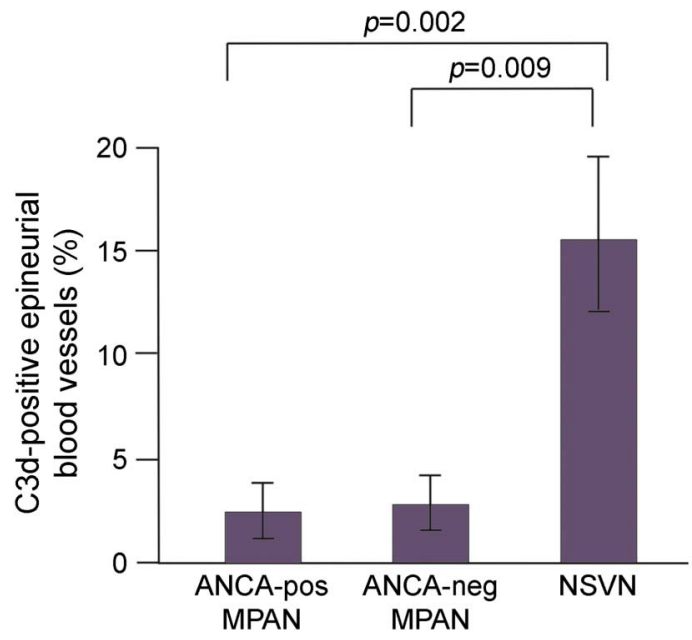

All epineurial blood vessels in the complete transverse sections of the sural nerve were assessed in each case. Error bars represent SEM. The percentages of C3d-positive epineurial blood vessels in the complete transverse sections of the sural nerve were $2.6 \pm 7.2$ in the antineutrophil cytoplasmic antibody (ANCA)-positive microscopic polyangiitisassociated neuropathy (MPAN) group, $3.1 \pm 5.2$ in the ANCA-negative MPAN group, and $15.7 \pm 18.0$ in the nonsystemic vasculitic neuropathy (NSVN) group ( $p=$ 0.001 , 1-way analysis of variance). The Tukey-Kramer method revealed significant differences between the ANCA-positive MPAN group and the NSVN group ( $p=$ 0.002 ), as well as between the ANCA-negative MPAN group and the NSVN group ( $p=0.009$ ). No significant difference was seen between the ANCA-positive and ANCA-negative MPAN groups. 

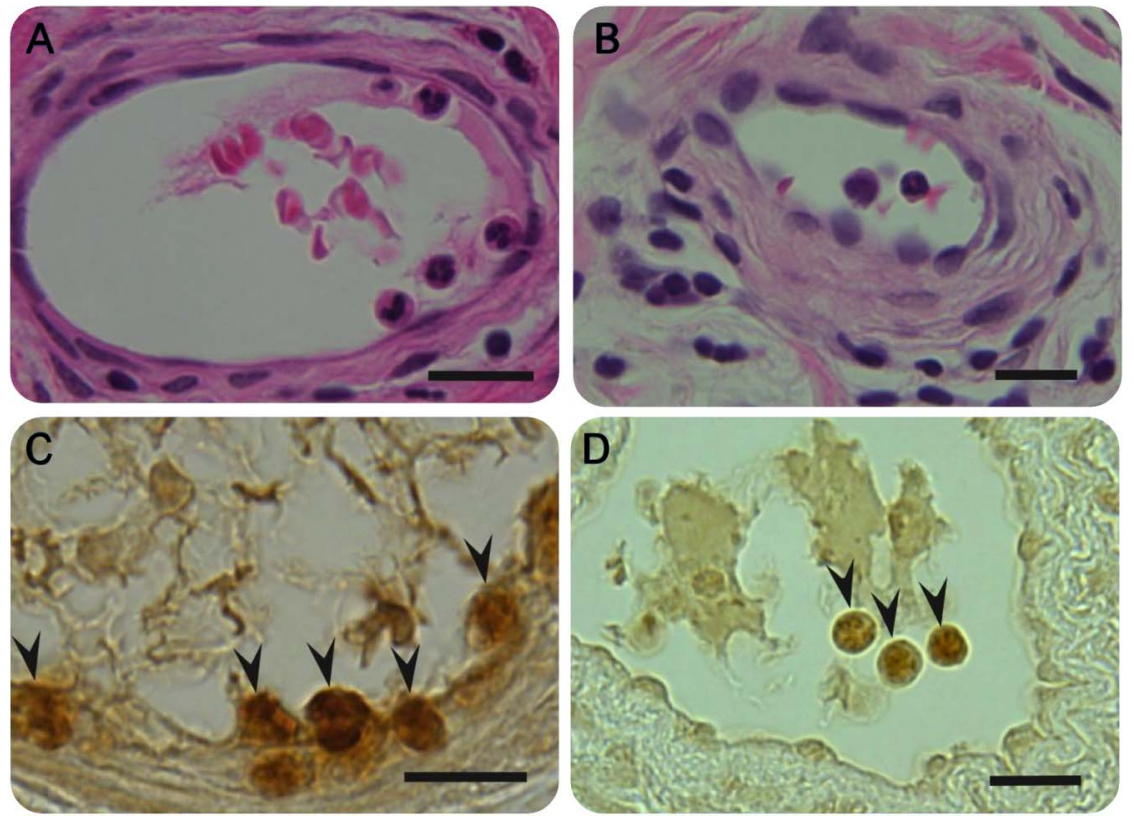

Photographs of microscopic polyangiitis-associated neuropathy (MPAN) (A and C) and nonsystemic vasculitic neuropathy (NSVN) (B and D). Hematoxylin and eosin staining (A and B) and myeloperoxidase (MPO) immunohistochemistry (C and D). Attachment of polymorphonuclear cells, which were considered neutrophils, to the endothelial cells of epineurial small vessels was frequently found in the MPAN groups, irrespective of the presence or absence of antineutrophil cytoplasmic antibodies (A and C), whereas such attachment was scarce in the NSVN group, although polymorphonuclear cells were present in vessel lumina (B and D). MPO-positive cells are indicated by arrowheads. Scale bars $=20 \mu \mathrm{m}$.

2 , digestive tract in 1 , and skin in 3 . Another case showed renal and pulmonary damage in addition to neuropathy. ESRs in these cases were variable, ranging from 35 to $120 \mathrm{~mm} / \mathrm{h}$.

Adhesion of neutrophils to epineurial vessels. On routine hematoxylin and eosin-stained sections, polymorphonuclear cells were occasionally detected in the lumina of epineurial small vessels in both the NSVN and MPAN groups. Because the majority of these cells had numerous small granules, these cells were considered to be neutrophils. ${ }^{24}$ Polymorphonuclear cells with relatively large eosinophilic granules, that are considered to be eosinophils, ${ }^{24}$ were also found in vessel lumina, although the number was much lower than neutrophils in both the NSVN and MPAN groups. Attachment of neutrophils to the endothelial cells of epineurial small vessels was frequently observed in the MPAN groups, irrespective of the presence or absence of ANCA, whereas such attachment was scarce in the NSVN group, although polymorphonuclear cells were present in vessel lumina (figure 3, A-D).

Under electron microscopy, neutrophils closely apposed to vascular endothelial cells without a gap were observed in MPAN cases, in accordance with light microscopic observation (figure 4, A and B). Some of the endothelial cells apposed to these neutrophils had numerous cytoplasmic organelles. These findings were observed in both ANCApositive MPAN and ANCA-negative MPAN groups.

Quantitative analysis was performed by immunohistochemistry with anti-MPO antibodies. Both neutrophils and monocytes were found to be MPO positive, but light and electron microscopy revealed that monocytes rarely attached to the endothelial cells of epineurial blood vessels in either NSVN or MPAN. The numbers of MPO-positive cells that attached to the endothelial cells of epineurial blood vessels were $0.12 \pm 0.08$ per epineurial vessel in the ANCA-positive MPAN group, $0.09 \pm 0.08$ in the ANCA-negative MPAN group, and $0.02 \pm 0.04$ in the NSVN group $(p<0.001$, figure 5 and table e-2). Because blood examination revealed higher white blood cell counts in the MPA groups than in the NSVN group, the result was verified by comparing the indices of the number of MPO-positive cells attached to the endothelial cells divided by the bold white blood cell count. It was still significant after this correction $\left(1.14 \times 10^{-3} \pm 0.89 \times 10^{-3}\right.$ in the ANCA-positive MPAN, $0.81 \times 10^{-3} \pm 0.72 \times$ $10^{-3}$ in the ANCA-negative MPAN, and $0.34 \times$ $10^{-3} \pm 0.45 \times 10^{-3}$ in the NSVN groups; $p=$ 0.002). Together, these findings suggest that vascular endothelial cell damage by neutrophils is a pathologic 
Figure 4 Neutrophil attachment to the vascular endothelium in patients with microscopic polyangiitis-associated neuropathy
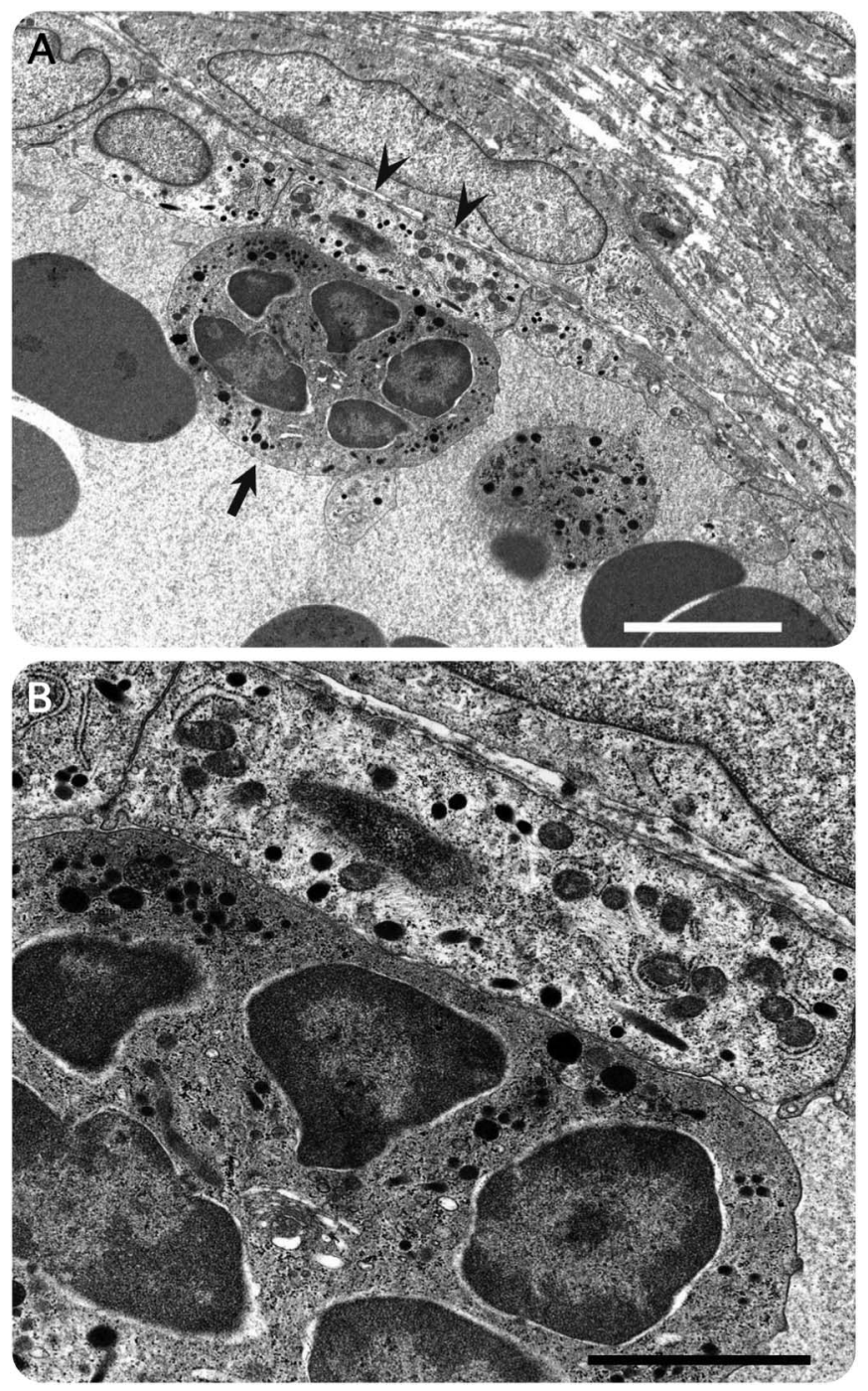

Uranyl acetate and lead citrate staining. Under electron microscopy, polymorphonuclear cells (arrow) closely apposed to endothelial cells (arrowhead) without a gap were observed. A high-powered view of $A$ is shown in B. Because these cells had numerous small granules of varying shapes in their cytoplasm, they were morphologically confirmed as being neutrophils. Some of the endothelial cells apposed to these neutrophils had numerous cytoplasmic organelles. Scale bars $=5 \mu \mathrm{m}(\mathrm{A})$ and $2 \mu \mathrm{m}(\mathrm{B})$.

signature of MPAN but not NSVN. Two ANCApositive patients without apparent involvement of other organs also had attachment of MPO-positive cells to the vascular endothelium ( 0.31 and 0.09 cells per epineurial vessel). Vascular C3d deposition was not observed in those patients.

Most patients with NSVN had normal or only mildly abnormal laboratory findings, but 1 had significantly elevated inflammatory indices (ESR $76 \mathrm{~mm} / \mathrm{h}$ and CRP $12.7 \mathrm{mg} / \mathrm{dL}$ ), with no findings suggestive of other organ involvement. This patient also had increased endothelial attachment of MPO-positive cells $(0.18$ cells per epineurial vessel) and negative C3d antibody staining.
DISCUSSION NSVN has been proposed by neurologists to be a neuropathy confined to the peripheral nervous system. ${ }^{8,11,13}$ It is one of the most common causes of vasculitic neuropathy, along with MPA and eosinophilic granulomatosis with polyangiitis. ${ }^{11,13,14,25}$ Whether this disease is truly an isolated nosological entity or simply a continuum on the spectrum of systemic vasculitides, particularly small vessel vasculitis including MPA, remains controversial., ${ }^{5,11}$ The ambiguity of the definition of NSVN seems to result from the absence of specific biomarkers, including autoantibodies. We compared the clinicopathologic features of MPA and NSVN, focusing on immune mechanisms that might trigger vasculitis, and clearly demonstrated differences between these 2 entities. The most notable finding in our study is that C3d deposition was frequently observed at epineurial small vessels in NSVN. Although antibodies triggering complement pathways were not clarified, this finding suggests that the complements participate in the pathogenesis of NSVN but not MPA.

As for the pathogenesis of MPA, attachment of neutrophils activated by ANCA to endothelial cells of small vessels is considered to be an initial trigger of vasculitis. ${ }^{26}$ This effect is also clearly demonstrated in our study, which showed this finding in both ANCA-positive and ANCA-negative cases classified as MPA. Regarding complement deposition in the pathogenesis of vasculitis, CHCC2012 nomenclature defines small vessel vasculitides with the participation of complement. ${ }^{1}$ However, the CHCC2012 nomenclature does not clearly distinguish NSVN. ${ }^{1}$ Therefore, the active participation of researchers in the field of neurology, particularly peripheral neuropathy, in vasculitis research is needed to establish the nosology of NSVN.

According to the initial report, the features of NSVN are defined as follows: (1) clinically, only nerves are affected; and (2) no or few constitutional symptoms or serologic abnormalities are present. $^{8}$ More recent guidelines for NSVN, published on behalf of the Peripheral Nerve Society, seem to adopt looser criteria, as they do not exclude patients with constitutional symptoms, such as fever, weight loss, malaise, fatigue, myalgias, and arthralgias. ${ }^{13}$ As for laboratory data indicating the severity of inflammation, the criteria included patients with an ESR less than $100 \mathrm{~mm} / \mathrm{h} \cdot{ }^{13}$ In addition, vasculitis detected in muscle biopsy specimens is not considered for exclusion. ${ }^{13}$ In this study, we included patients in the NSVN group based on these criteria. Most patients with NSVN had normal or only mildly abnormal laboratory findings, but 1 had elevated inflammatory indices, increased endothelial attachment of neutrophils, and negative C3d antibody staining as described in the Results section. This patient may 
Figure 5 The degree of attachment of myeloperoxidase-positive cells to epineurial vessels

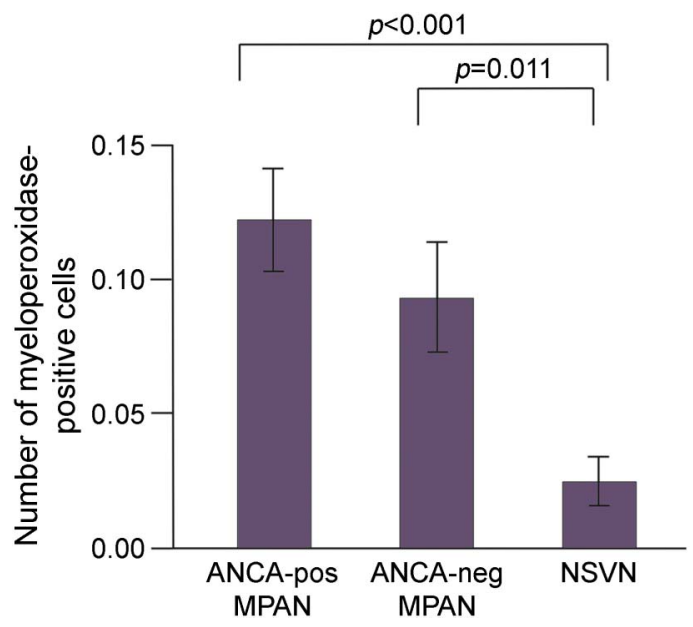

All epineurial blood vessels in the complete transverse sections of the sural nerve were assessed in each case. Error bars represent the SEM. The numbers of myeloperoxidase-positive cells attached to the endothelial cells of epineurial blood vessels were $0.12 \pm 0.08$ per epineurial vessel in the antineutrophil cytoplasmic antibody (ANCA)positive microscopic polyangiitis-associated neuropathy (MPAN) group, $0.09 \pm 0.08$ in the ANCA-negative MPAN group, and $0.02 \pm 0.04$ in the nonsystemic vasculitic neuropathy (NSVN) group ( $<0.001$, 1-way analysis of variance). The Tukey-Kramer method revealed significant differences between the ANCA-positive MPAN group and the NSVN group ( $p<0.001$ ), as well as between the ANCAnegative MPAN group and the NSVN group $(p=0.011)$. No significant difference was seen between the ANCA-positive and ANCA-negative MPAN groups.

have had a pathogenesis similar to MPA rather than NSVN.

Some patients with MPA may initially present with characteristics indistinguishable from those of NSVN. One patient, who was ultimately diagnosed with MPA, manifested only symptoms suggestive of peripheral nervous system involvement and not systemic involvement for 2 years. Sural nerve biopsy was performed when the patient manifested a recurrence of neuropathy and systemic involvement involving the skin and gastrointestinal tract. Another patient included in the MPAN group was initially diagnosed with NSVN at the time of nerve biopsy, but serum ANCA became positive 4 years later. At the time of nerve biopsy, ESR $(70 \mathrm{~mm} / \mathrm{h})$ and CRP $(12.0 \mathrm{mg} / \mathrm{dL})$ were high compared with most patients with NSVN. The specimens from these patients showed attachment of neutrophils to the endothelial cells of small epineurial vessels and negative C3d staining, which are characteristic of MPA, despite the initial diagnosis of NSVN.

Currently, the most commonly used nomenclature for vasculitides is CHCC2012, which was originally published in 1994 to propose names and definitions for the most common forms of vasculitis, including MPA. ${ }^{1,27}$ According to this nomenclature, MPA is defined as vasculitis that affects small blood vessels with few or no immune deposits and is often associated with ANCA. ${ }^{1}$ As MPA does not have specific clinical or laboratory characteristics, such as the presence of asthma or eosinophilia in eosinophilic granulomatosis with polyangiitis or granulomatous inflammation in the respiratory tract in granulomatosis with polyangiitis, the diagnosis of MPA depends on the exclusion of other diseases unless immune deposits are evaluated. Although CHCC2012 included the concept of single-organ vasculitis, such as cutaneous small vessel vasculitis, testicular arteritis, and CNS vasculitis, vasculitis confined to the peripheral nervous system was not mentioned. ${ }^{1}$ Therefore, the distinction between MPA and NSVN has not yet been clarified based on current nomenclature. As this study suggests that NSVN and MPA are distinct with respect to the mechanism of vasculitis, establishing the position of NSVN in the spectrum of vasculitides on the basis of CHCC2012 nomenclature is needed. The data support targeted therapies based on the mechanisms of NSVN. For example, eculizumab, a recombinant humanized monoclonal antibody that inhibits complement cascades, may be effective in patients with NSVN. Further studies regarding the search for specific biomarkers, such as causative autoantibodies that trigger complement cascades, are also needed to clarify the mechanisms of vasculitis in NSVN.

\section{AUTHOR CONTRIBUTIONS}

Haruki Koike and Gen Sobue developed the hypotheses and conceived the study. Mie Takahashi, Haruki Koike, Shohei Ikeda, Yuichi Kawagashira, Masahiro Iijima, Atsushi Hashizume, and Masahisa Katsuno compiled and analyzed the clinical data. Mie Takahashi, Haruki Koike, Shohei Ikeda, Yuichi Kawagashira, Masahiro Iijima, and Gen Sobue performed the pathologic analysis. Gen Sobue supervised the study. Haruki Koike wrote the first draft, and all authors critically evaluated the manuscript.

\section{STUDY FUNDING}

This work was supported by grants from the Ministry of Health, Labor and Welfare (Research on rare and intractable diseases, H26-057) and the Ministry of Education, Culture, Sports, Science and Technology (25461276) of Japan.

\section{DISCLOSURE}

M. Takahashi reports no disclosures. H. Koike received research support from the Ministry of Education, Culture, Sports, Science and Technology of Japan and the Ministry of Health, Welfare and Labor of Japan. S. Ikeda, Y. Kawagashira, M. Iijima, and A. Hashizume report no disclosures. M. Katsuno served on the scientific advisory board for Pharmaceuticals and medical devise agency, Japan; received travel funding and speaker honoraria from Tanabe-Mitsubishi; consulted for Riprocel, Asbio Pharma; holds a patent for nonhuman animal reproducing pathogenic conditions of spinal and bulbar muscular atrophy and remedy for spinal and bulbar muscular atrophy and Anti-spinal and bulbar muscular atrophy; and received research support from the Ministry of Education, Culture, Sports, Science and Technology of Japan and the Japan Agency for 
Medical Research and Development. G. Sobue served on the scientific advisory board for the Kanae Science Foundation for the Promotion of Medical Science, the Naito Science Foundation, and the Takeda Foundation; served on the editorial board for Brain, Degenerative Neurological and Neuromuscular Disease, Journal of Neurology, Amyotrophic Lateral Sclerosis; and received research support from the Ministry of Education, Culture, Sports, Science and Technology of Japan, the Ministry of Welfare, Health and Labor of Japan, The Japan Science and Technology Agency, and Core Research for Evolutional Science and Technology. Go to Neurology.org/nn for full disclosure forms.

Received August 16, 2017. Accepted in final form September 7, 2017.

\section{REFERENCES}

1. Jennette JC, Falk RJ, Bacon PA, et al. 2012 revised international Chapel Hill consensus conference nomenclature of vasculitides. Arthritis Rheum 2013;65:1-11.

2. Guillevin L, Cohen P, Gayraud M, Lhote F, Jarrousse B, Casassus P. Churg-Strauss syndrome. Clinical study and long-term follow-up of 96 patients. Medicine (Baltimore) 1999;78:26-37.

3. Guillevin L, Durand-Gasselin B, Cevallos R, et al. Microscopic polyangiitis: clinical and laboratory findings in eighty-five patients. Arthritis Rheum 1999;42:421-430.

4. Nukada H, Dyck PJ. Microsphere embolization of nerve capillaries and fiber degeneration. Am J Pathol 1984;115: 275-287.

5. Morozumi S, Koike H, Tomita M, et al. Spatial distribution of nerve fiber pathology and vasculitis in microscopic polyangiitis-associated neuropathy. J Neuropathol Exp Neurol 2011;70:340-348.

6. Hattori N, Ichimura M, Aoki S, et al. Clinicopathological features of Churg-Strauss syndrome-associated neuropathy. Brain 1999;122:427-439.

7. Kissel JT, Slivka AP, Warmolts JR, Mendell JR. The clinical spectrum of necrotizing angiopathy of the peripheral nervous system. Ann Neurol 1985;18:251-257.

8. Dyck PJ, Benstead TJ, Conn DL, Stevens JC, Windebank AJ, Low PA. Nonsystemic vasculitic neuropathy. Brain 1987;110:843-853

9. Said G, Lacroix-Ciaudo C, Fujimura H, Blas C, Faux N. The peripheral neuropathy of necrotizing arteritis: a clinicopathological study. Ann Neurol 1988;23:461-465.

10. Davies L, Spies JM, Pollard JD, McLeod JG. Vasculitis confined to peripheral nerves. Brain 1996;119 1441-1448.

11. Sugiura M, Koike H, Iijima M, et al. Clinicopathologic features of nonsystemic vasculitic neuropathy and microscopic polyangiitis-associated neuropathy: a comparative study. J Neurol Sci 2006;241:31-37.

12. Collins MP, Periquet MI, Mendell JR, Sahenk Z, Nagaraja HN, Kissel JT. Nonsystemic vasculitic neuropathy: insights from a clinical cohort. Neurology 2003;61: 623-630.
13. Collins MP, Dyck PJ, Gronseth GS, et al. Peripheral Nerve Society Guideline on the classification, diagnosis, investigation, and immunosuppressive therapy of nonsystemic vasculitic neuropathy: executive summary. J Peripher Nerv Syst 2010;15:176-184.

14. Hattori N, Mori K, Misu K, Koike H, Ichimura M, Sobue G. Mortality and morbidity in peripheral neuropathy associated Churg-Strauss syndrome and microscopic polyangiitis. J Rheumatol 2002;29:1408-1414.

15. Koike H, Iijima M, Sugiura M, et al. Alcoholic neuropathy is clinicopathologically distinct form thiamine-deficiency neuropathy. Ann Neurol 2003;54:19-29.

16. Koike H, Hirayama M, Yamamoto M, et al. Age associated axonal features in HNPP with 17p11.2 deletion in Japan. J Neurol Neurosurg Psychiatry 2005; 76 : 1109-1114.

17. Koike H, Atsuta N, Adachi H, et al. Clinicopathological features of acute autonomic and sensory neuropathy. Brain 2010;133:2881-2896.

18. Kawagashira Y, Koike H, Tomita M, et al. Morphological progression of myelin abnormalities in IgM-monoclonal gammopathy of undetermined significance anti-myelinassociated glycoprotein neuropathy. J Neuropathol Exp Neurol 2010;69:1143-1157.

19. Hashimoto R, Koike H, Takahashi M, et al. Uncompacted myelin lamellae and nodal ion channel disruption in POEMS syndrome. J Neuropathol Exp Neurol 2015;74: 1127-1136.

20. Koike H, Kadoya M, Kaida KI, et al. Paranodal dissection in chronic inflammatory demyelinating polyneuropathy with anti-neurofascin-155 and anti-contactin-1 antibodies. J Neurol Neurosurg Psychiatry 2017;88:465-473.

21. Dyck PJ, Dyck PJB, Engelstad J. Pathologic alterations of nerves. In: Dyck PJ, Thomas PK, editors. Peripheral Neuropathy. 4th ed. Philadelphia: Elsevier Saunders; 2005: 733-829.

22. Koike H, Iijima M, Mori K, et al. Neuropathic pain correlates with myelinated fibre loss and cytokine profile in POEMS syndrome. J Neurol Neurosurg Psychiatry 2008; 79:1171-1179.

23. Ohyama K, Koike H, Takahashi M, et al. Immunoglobulin G4-related pathologic features in inflammatory neuropathies. Neurology 2015;85:1400-1407.

24. Secchi J, Lecaque D. Tissues. In: Histology Atlas. Paris: Maloine S.A.; 1981:31-70.

25. Koike H, Sobue G. Clinicopathological features of neuropathy in anti-neutrophil cytoplasmic antibody-associated vasculitis. Clin Exp Nephrol 2013;17:683-685.

26. Jarrot PA, Kaplanski G. Pathogenesis of ANCA-associated vasculitis: an update. Autoimmun Rev 2016;15:704-713.

27. Jennette JC, Falk RJ, Andrassy K, et al. Nomenclature of systemic vasculitides. Proposal of an international consensus conference. Arthritis Rheum 1994;37:187-192. 


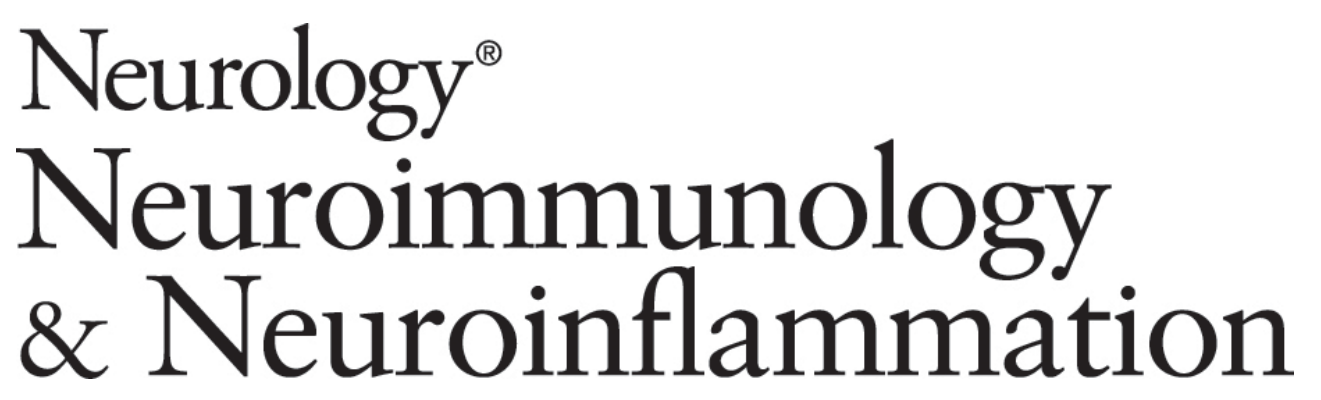

Distinct pathogenesis in nonsystemic vasculitic neuropathy and microscopic polyangiitis

Mie Takahashi, Haruki Koike, Shohei Ikeda, et al.

Neurol Neuroimmunol Neuroinflamm 2017;4;

DOI 10.1212/NXI.0000000000000407

This information is current as of October 23, 2017

Neurol Neuroimmunol Neuroinflamm is an official journal of the American Academy of Neurology.

Published since April 2014, it is an open-access, online-only, continuous publication journal. Copyright

Copyright $\odot 2017$ The Author(s). Published by Wolters Kluwer Health, Inc. on behalf of the American

Academy of Neurology.. All rights reserved. Online ISSN: 2332-7812.

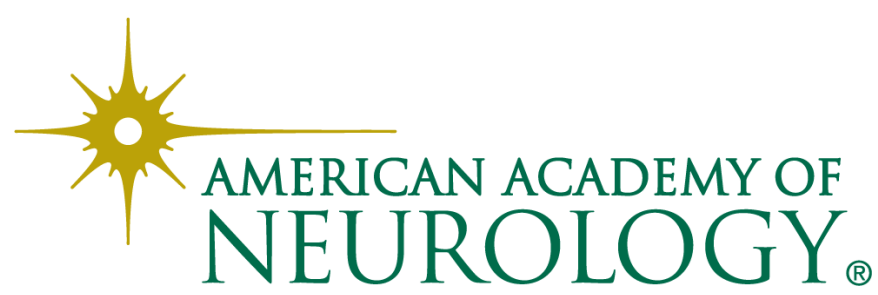




\section{Updated Information \& Services}

\section{Supplementary Material}

\section{References}

Citations

Subspecialty Collections

Permissions \& Licensing

Reprints including high resolution figures, can be found at: http://nn.neurology.org/content/4/6/e407.full.html

Supplementary material can be found at: http://nn.neurology.org/content/suppl/2017/11/06/4.6.e407.DC1

This article cites 25 articles, 4 of which you can access for free at: http://nn.neurology.org/content/4/6/e407.full.html\#\#ref-list-1

This article has been cited by 1 HighWire-hosted articles: http://nn.neurology.org/content/4/6/e407.full.html\#\#otherarticles

This article, along with others on similar topics, appears in the following collection(s):

\section{Peripheral neuropathy}

http://nn.neurology.org//cgi/collection/peripheral_neuropathy

\section{Vasculitis}

http://nn.neurology.org//cgi/collection/vasculitis

Information about reproducing this article in parts (figures,tables) or in its entirety can be found online at:

http://nn.neurology.org/misc/about.xhtml\#permissions

Information about ordering reprints can be found online: http://nn.neurology.org/misc/addir.xhtml\#reprintsus

Neurol Neuroimmunol Neuroinflamm is an official journal of the American Academy of Neurology.

Published since April 2014, it is an open-access, online-only, continuous publication journal. Copyright

Copyright $\odot 2017$ The Author(s). Published by Wolters Kluwer Health, Inc. on behalf of the American Academy of Neurology.. All rights reserved. Online ISSN: 2332-7812.

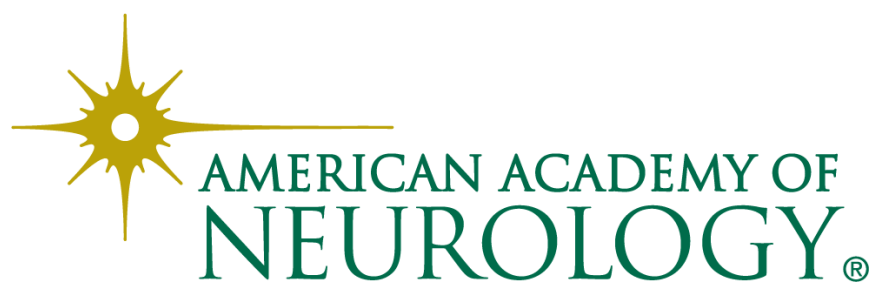

